\title{
Simple Vertices of Maximal Minor Polytopes*
}

\author{
Prakash Santhanakrishnan and Andrei Zelevinsky \\ Department of Mathematics, Northeastern University, \\ Boston, MA 02115, USA \\ andrei@northeastern.edu
}

\begin{abstract}
The maximal minor polytope $\Pi_{m, n}$ is the Newton polytope of the product of all maximal minors of an $m \times n$ matrix of indeterminates. The family of polytopes $\left\{\Pi_{m, n}\right\}$ interpolates between the symmetric transportation polytope (for $m=n-1$ ) and the permutohedron (for $m=2$ ). Both transportation polytope and the permutohedron are simple polytopes but in general $\Pi_{m, n}$ is not simple. The main result of this paper is an explicit construction of a class of simple vertices of $\Pi_{m, n}$ for general $m$ and $n$. We call them vertices of diagonal type. For every such vertex $v$ we explicitly describe all the edges and facets of $\Pi_{m, n}$ which contain $v$. Simple vertices of $\Pi_{m, n}$ have an interesting algebro-geometric application: they correspond to nonsingular extreme toric degenerations of the determinantal variety of $m \times n$ matrices not of full rank.
\end{abstract}

\section{Introduction}

In this paper we study the Newton polytope $\Pi_{m, n}$ of the product of all maximal minors of an $m \times n$ matrix of indeterminates, where $n>m \geq 2$. This is a lattice polytope in the space $\mathbf{R}^{m \times n}$ of real $m \times n$ matrices. The study of $\Pi_{m, n}$ had begun in [7], where it was shown that it appears naturally in various algebro-geometric and analytic contexts. In particular, it was conjectured in [7] that maximal minors form a universal Gröbner basis for their ideal, and this has been proven in [1] by exploring the combinatorial structure of vertices of $\Pi_{m, n}$.

The polytope $\Pi_{m, n}$ exhibits a remarkably rich combinatorial structure which is still far from being completely understood. Its dimension is equal to $(m-1)(n-1)$ but even this most basic fact was not given a direct proof in [7]: it was deduced from the interpretation of $\Pi_{m, n}$ as the Chow polytope of the

\footnotetext{
* Andrei Zelevinsky was partially supported by the NSF under Grant DMS-9104867.
} 
determinantal variety $\nabla_{m, n}=\left\{X \in \mathbf{C}^{m \times n}: \operatorname{rk}(X)<m\right\}$. A direct combinatorial proof of the equality $\operatorname{dim}\left(\Pi_{m, n}\right)=(m-1)(n-1)$ is a consequence of the technique developed in this present paper.

If $m=n-1$, then $\Pi_{n-1, n}$ is the transportation polytope of nonnegative $(n-1) \times n$ matrices having all row sums equal to $n$ and all column sums equal to $n-1\left[7\right.$, Theorem 2.8]. If $m=2$, then $\Pi_{2, n}$ is the permutohedron, which is the convex hull in $\mathbf{R}^{2 \times n}$ of all $n$ ! matrices obtained from

$$
\left(\begin{array}{ccccc}
n-1 & n-2 & \cdots & 1 & 0 \\
0 & 1 & \cdots & n-2 & n-1
\end{array}\right)
$$

by permuting columns [7, Proposition 1.12]. Thus the family $\left\{\Pi_{m, n}\right\}$ interpolates between the transportation polytope and the permutohedron.

Both transportation polytope and the permutohedron are known to be simple polytopes [5], [9], i.e., for each of them the edges going out of each vertex generate a simplicial cone. It was shown in [7] that the polytope $\Pi_{3,5}$ is also simple but $\Pi_{3,6}$ is not simple. The main result of this present paper is an explicit construction of a class of simple vertices of $\Pi_{m, n}$ for general $m$ and $n$. We call them vertices of diagonal type. For every such vertex $v$ we explicitly describe all the edges and facets of $\Pi_{m, n}$ which contain $v$. Simple vertices of $\Pi_{m, n}$ have an interesting algebro-geometric meaning: every such vertex corresponds to an extreme toric degeneration of $\nabla_{m, n}$ which represents a smooth point of the closure of the torus orbit of $\nabla_{m, n}$ on the Chow variety [4], [6, Theorem 1.10].

Our point of departure is the Support Theorem from [7]. It gives the characterization of all possible supports of vertices of $\Pi_{m, n}$ (the support of a matrix $\left(x_{i j}\right)$ is the set of indices $(i, j)$ such that $\left.x_{i j} \neq 0\right)$. Let $\Sigma \subset[m] \times[n]$ be a set of matrix indices (we use the standard abbreviation $[m]=\{1,2, \ldots, m\}$ ). We always express $\Sigma$ as $\Sigma=\bigcup_{i=1}^{m}\left(\{i\} \times J_{i}\right)$, where $J_{1}, \ldots, J_{m} \subset[n]$.

Support Theorem [7, Theorem 3.6]. Let $J_{1}, \ldots, J_{m}$ be subsets of $[n]$, and

$$
\Sigma=\bigcup_{i=1}^{m}\left(\{i\} \times J_{i}\right) \subset[m] \times[n]
$$

Then the following conditions are equivalent:

(a) There exists a vertex $v$ of $\Pi_{m, n}$ with $\operatorname{supp}(v)=\Sigma$.

(b) Each of the subsets $J_{1}, \ldots, J_{m}$ has cardinality $n-m+1$, and for every nonempty subset $\left\{i_{1}, i_{2}, \ldots, i_{p}\right\} \subseteq[m]$ we have

$$
\operatorname{card}\left(J_{i_{1}} \cup J_{i_{2}} \cup \cdots \cup J_{i_{p}}\right) \geq n-m+p
$$

A subset $\Sigma=\bigcup_{i=1}^{m}\left(\{i\} \times J_{i}\right)$ of $[m] \times[n]$ is called a support set for $\Pi_{m, n}$ if it satisfies the equivalent conditions of the Support Theorem. For every support set $\Sigma$ we define $\Pi_{m, n}(\Sigma)=\Pi_{m, n}\left(J_{1}, \ldots, J_{m}\right)$ to be the convex hull of all vertices of $\Pi_{m, n}$ 
with support $\Sigma$. It was proven in Proposition 3.7 of [7] that each $\Pi_{m, n}(\Sigma)$ is a face of $\Pi_{m, n}$. The faces of type $\Pi_{m, n}(\Sigma)$ are called the support faces of $\Pi_{m, n}$. They form a very interesting family of polytopes. For example, if $J_{i}=[n] \backslash[m] \cup\{i\}$ for $i \in[m]$, then $\Pi_{m, n}\left(J_{1}, \ldots, J_{m}\right)$ is the secondary polytope of the product of two simplices $\Delta^{m-1} \times \Delta^{n-m-1}$ [3] whose vertices correspond to regular triangulations of $\Delta^{m-1} \times \Delta^{n-m-1}$. We hope that other support faces of $\Pi_{m, n}$ also admit a nice geometric interpretation, and we feel that the study of the secondary polytope should benefit from its comparison with other support faces. One of the main motivations for this paper was to gain a better understanding of the structure of support faces.

It was conjectured in Conjecture 3.8 of [7] that all support faces of $\Pi_{m, n}$ have the same dimension $(m-1)(n-m-1)$. The technique developed here enables us to prove it for $n \leq 6$ (Proposition 5.8 below). In general, we prove only the inequality $\operatorname{dim}\left(\Pi_{m, n}(\Sigma)\right) \leq(m-1)(n-m-1)$ (Theorem 1.1 below). We establish the conjecture for some special class of supports, which we call supports of diagonal type. Such a support is specified by a sequence $c=\left(c_{1}, \ldots, c_{m-1}\right)$ of distinct indices in $[n]$. It is defined by the following construction. We define successively

$$
\begin{array}{cc}
J_{1}=J_{1}(c)=[n] \backslash\left\{c_{1}, c_{2}, c_{3}, \ldots, c_{m-1}\right\}, & \mu_{1}=\min J_{1}(c), \\
J_{2}=J_{2}(c)=[n] \backslash\left\{\mu_{1}, c_{2}, c_{3}, \ldots, c_{m-1}\right\}, & \mu_{2}=\min J_{2}(c), \\
J_{3}=J_{3}(c)=[n] \backslash\left\{\mu_{1}, \mu_{2}, c_{3}, \ldots, c_{m-1}\right\}, & \mu_{3}=\min J_{3}(c), \\
\vdots & \\
J_{m}=J_{m}(c)=[n] \backslash\left\{\mu_{1}, \mu_{2}, \mu_{3}, \ldots, \mu_{m-1}\right\} .
\end{array}
$$

It is easy to see that $J_{1}, \ldots, J_{m}$ are well defined and satisfy the conditions of the Support Theorem. We will construct a simple vertex $v=v(c)$ of diagonal type for every support given by the above construction. The equality

$$
\operatorname{dim} \Pi_{m, n}\left(J_{1}(c), \ldots, J_{m}(c)\right)=(m-1)(n-m-1)
$$

will result immediately from the description of the edges of $\Pi_{m, n}$ containing $v(c)$.

According to [7], the vertices of $\Pi_{m, n}$ are combinatorially encoded by coherent matching fields. For every $m$-element subset $\sigma \in[n]$ a matching with support $\sigma$ is a bijection $\Lambda_{\sigma}: \sigma \rightarrow[m]$. By slight abuse of notation, we use the same symbol $\Lambda_{\sigma}$ for the graph of a matching $\left\{(i, j) \in[m] \times[n]: j \in \sigma, i=\Lambda_{\sigma}(j)\right\}$, and also for its indicator matrix. A matching field of format $m \times n$ is a choice of a matching $\Lambda_{\sigma}$ for each $m$-element subset $\sigma \subset[n]$. Given any matching field $\Lambda=\left(\Lambda_{\sigma}\right)$, we let $v(\Lambda)=\sum_{\sigma} \Lambda_{\sigma}$ denote the sum of indicator matrices of all matchings $\Lambda_{\sigma}$. The polytope $\Pi_{m, n}$ can be combinatorially defined as the convex hull in $\mathbf{R}^{m \times n}$ of the matrices $v(\Lambda)$ for all matching fields $\Lambda$ (see Section 1 of [7]). A matching field $\Lambda$ is called coherent if $v(\Lambda)$ is a vertex of the polytope $\Pi_{m, n}$. 
The coherent matching field $\Lambda=\left(\Lambda_{\sigma}\right)$ encoding the vertex $v(c)$ of diagonal type can be defined as follows: for every m-element subset $\sigma \subset[n]$ the matching $\Lambda_{a}$ is lexicographically first among all the matchings with support $\sigma$ which are contained in $\bigcup_{i=1}^{m}\left(\{i\} \times J_{i}(c)\right)$. Here the lexicographic order for matchings with given support is defined so that a matching $\left\{\left(1, j_{1}\right),\left(2, j_{2}\right), \ldots,\left(m, j_{m}\right)\right\}$ precedes

$$
\left\{\left(1, j_{1}^{\prime}\right),\left(2, j_{2}^{\prime}\right), \ldots,\left(m, j_{m}^{\prime}\right)\right\}
$$

if the first nonzero difference $j_{m}^{\prime}-j_{m}$ is positive. The main technical tool in our analysis of $v(c)$ is the fact that $\Lambda$ is also given by an appropriate version of the greedy algorithm.

We can describe explicitly all the facets of $\Pi_{m, n}$ containing vertices of diagonal type. First, there are $m n$ simplest facets supported by the hyperplanes $\left\{x_{i j}=0\right\}$ (by definition, the support faces are minimal intersections of such facets). To describe the remaining facets we need some terminology and notation. For $1 \leq k \leq m, 1 \leq r \leq n$ we put

$$
b_{k r}=\sum_{t} \min (k, t)\left(\begin{array}{l}
r \\
t
\end{array}\right)\left(\begin{array}{l}
n-r \\
m-t
\end{array}\right)
$$

A pair of subsets $I \subset[m], J \subset[n]$ is balanced if $1 \leq \operatorname{card}(I) \leq m-1,1 \leq$ $\operatorname{card}(J)-\operatorname{card}(I) \leq n-m-1$; in other words, this means that $1 \leq \operatorname{card}(I)<$ $\operatorname{card}(J)$ and $1 \leq \operatorname{card}(\bar{I})<\operatorname{card}(\bar{J})$, where $\bar{I}=[m] \backslash I, \bar{J}=[n] \backslash J$. Our analysis of vertices of diagonal type implies the following.

Theorem 0.1. For every balanced pair $(I, J)$ with $\operatorname{card}(I)=k, \operatorname{card}(J)=r$ the hyperplane $\left\{\sum_{i \in I, j \in J} x_{i j}=b_{k r}\right\}$ in $\mathbf{R}^{m \times n}$ supports a facet of $\Pi_{m, n}$.

We call the facets from Theorem 0.1 simple. We say that a vertex of $\Pi_{m, n}$ is very simple if it lies exactly on $(m-1)(n-m-1)$ simple facets of $\Pi_{m, n}$ and on $m(m-1)$ facets of type $\left\{x_{i j}=0\right\}$, and it does not belong to any other facet. Clearly, every very simple vertex of $\Pi_{m, n}$ is a simple vertex of $\Pi_{m, n}$, and also a simple vertex of its support face. We conclude that if a support face $\Gamma$ of $\Pi_{m, n}$ contains a very simple vertex, then $\operatorname{dim}(\Gamma)=(m-1)(n-m-1)$.

Theorem 0.2. Every vertex of $\Pi_{m, n}$ of diagonal type is very simple.

Theorems 0.1 and 0.2 suggest the following way of thinking about $\Pi_{m, n}$. Let $T_{m, n}$ denote the transportation polytope of nonnegative $m \times n$ matrices having all row sums equal to $\left(\begin{array}{l}n \\ m\end{array}\right)$ and all column sums equal to $\left(\begin{array}{c}n-1 \\ m-1\end{array}\right)$. Clearly, $\Pi_{m, n} \subseteq T_{m, n}$, and both polytopes have the same dimension $(m-1)(n-1)$. The polytope $T_{m, n}$ can be viewed as the "first approximation" to $\Pi_{m, n}$. Now consider the subpolytope $S_{m, n} \subset T_{m, n}$ defined by additional constraints $\left\{\sum_{i \in I, j \in J} x_{i j} \leq b_{k r}\right\}$, where $(I, J)$ runs over all balanced pairs from Theorem 0.1. By Theorem 0.1 , 
$\Pi_{m, n} \subseteq S_{m, n} \subseteq T_{m, n}$. We view $S_{m, n}$ as the "second approximation" to $\Pi_{m, n}$. In some interesting special cases this approximation is exact, i.e., $\Pi_{m, n}=S_{m, n}$. This is true for the permutohedron $\Pi_{2, n}$ (see, e.g., [9]) and for $\Pi_{3,5}$ [7]. Now Theorem 0.2 says that every simple vertex of $\Pi_{m, n}$ of diagonal type is also a vertex of $S_{m, n}$. It is conceivable that this is true for all simple vertices of $\Pi_{m, n}$. It would be interesting to study the polytope $S_{m, n}$ in more detail, in particular to classify its vertices and/or its simple vertices. For example, it seems plausible that the Support Theorem remains true for $S_{m, n}$.

The class of supports and vertices of diagonal type is less restrictive than it seems. Our polytope $\Pi_{m, n}$ has a big group of symmetries $S_{m} \times S_{n}$ acting by permutations of rows and columns, and the statements such as simplicity of a vertex, or the value of the dimension of a support set are preserved by these symmetries. It turns out that diagonal and pointed diagonal matching fields from [7] give rise to vertices of diagonal type. These and other examples are treated in Section 5. Still for general $m$ and $n$ not every support set can be obtained from a support of diagonal type by permutations of rows and columns.

The notion of the lexicographically first matching field makes sense for arbitrary support sets, not only for supports of diagonal type. It would be interesting to classify support sets such that the corresponding lexicographically first matching field defines a very simple vertex. We prove that if $n \leq 6$, then every support set can be transformed into such a position by permutations of rows and columns (Proposition 5.8 below).

The material of this paper is organized as follows. In Section 1 we prove that the dimension of every support face of $\Pi_{m, n}$ is not greater than $(m-1)(n-m-1)$ (Theorem 1.1). Sections 2 and 3 contain some technical preparations. In Section 4 we describe the normal cones of $\Pi_{m, n}$ at vertices of diagonal type. Examples are discussed in Section 5.

\section{Dimensions of Support Faces: An Upper Bound}

In this section we prove the following result.

Theorem 1.1. The dimension of every support face of $\Pi_{m, n}$ is not greater than $(m-1)(n-m-1)$.

Proof. As already mentioned in the Introduction, the polytope $\Pi_{m, n}$ lies in the affine subspace of $\mathbf{R}^{m \times n}$ consisting of matrices having all row sums equal to $\left(\begin{array}{l}n \\ m\end{array}\right)$ and all column sums equal to $\left(\begin{array}{l}n-1 \\ m-1\end{array}\right)$. Let $E \subset \mathbf{R}^{m \times n}$ denote the vector subspace of matrices with all row and column sums equal to 0 . Then all the edges of $\Pi_{m, n}$ belong to $E$. We have $\operatorname{codim}(E)=m+n-1$ hence $\operatorname{dim}(E)=(m-1)(n-1)$. To see this consider the linear map $\mathbf{R}^{m \times n} \rightarrow \mathbf{R}^{m} \times \mathbf{R}^{n}$ taking a matrix $\left(x_{i j}\right)$ to the vector $(x, y)$ given by $x_{i}=\sum_{j} x_{i j}, y_{j}=-\sum_{i} x_{i j}$. Clearly, this map identifies $\mathbf{R}^{m \times n} / E$ with the hyperplane $\left\{(x, y): \sum_{i} x_{i}+\sum_{j} y_{j}=0\right\}$ in $\mathbf{R}^{m} \times \mathbf{R}^{n}$. 
Now let $J_{1}, \ldots, J_{m} \subset[n]$ be subsets satisfying the conditions of part (b) of the Support Theorem, and let $\Gamma=\Pi_{m, n}\left(J_{1}, \ldots, J_{m}\right)$ be the corresponding support face of $\Pi_{m, n}$. Let $\bar{J}_{i}:=[n] \backslash J_{i}$. By definition, all the edges of $\Gamma$ lie in the vector space $E(\Gamma):=E \cap\left\{x_{i j}=0\right.$ for $\left.i \in[m], j \in \bar{J}_{i}\right\}$. Therefore, to prove Theorem 1.1 it is enough to show that $\operatorname{dim}(E(\Gamma))=(m-1)(n-m-1)$ or, equivalently, that

$$
\operatorname{codim}_{E}(E(\Gamma))=m(m-1)
$$

For a matrix index $(i, j)$ let $\varphi_{i j} \in E^{*}$ be the linear form on $E$ sending each matrix $\left(x_{i j}\right) \in E$ to its entry $x_{i j}$. Since $E(\Gamma)$ as a subspace of $E$ is defined by $m(m-1)$ linear equations, it is enough to show that all $\varphi_{i j}$ for $i \in[m], j \in \bar{J}_{i}$ are linearly independent. To this end, we represent any subset $\Sigma \subset[m] \times[n]$ of matrix indices by means of the bipartite graph $G(\Sigma)$ with vertices labeled by row and column indices, where a row $i$ is joined by an edge with a column $j$ if and only if $(i, j) \in \Sigma$. We use the following combinatorial criterion for linear independence.

Lemma 1.2. The following conditions on a subset $\Omega \subset[m] \times[n]$ are equivalent:

(a) The linear forms $\left\{\varphi_{i j}:(i, j) \in \Omega\right\}$ are linearly independent.

(b) The bipartite graph $G(\bar{\Omega})$ corresponding to $\bar{\Omega}:=([m] \times[n]) \backslash \Omega$ is connected.

Proof. The proof is an exercise in matroid theory. Consider the matroid $\mathscr{M}$ on the set $[m] \times[n]$ defined by the family of linear forms $\left\{\varphi_{i j}: i \in[m], j \in[n]\right\}$ on $E$. By definition, the dual matroid $\mathscr{M}^{*}$ is defined by the images of standard basis vectors of $\mathbf{R}^{m \times n}$ (i.e., matrix units $E_{i j}$ ) in the quotient space $\mathbf{R}^{m \times n} / E$. Using the projection $\mathbf{R}^{m \times n} \rightarrow \mathbf{R}^{m} \times \mathbf{R}^{n}$ described above, we identify $\mathscr{M}^{*}$ with the cycle matroid of the full bipartite graph $K_{m n}$ (see, e.g., 1.10 and 9.5 of [8]). By definition of the dual matroid, a subset $\Omega \subset[m] \times[n]$ is independent in $\mathscr{M}$ if and only if $\Omega$ has full rank in $\mathscr{M}^{*}$. By $1.10(1)$ of [8], the latter condition is equivalent to (b).

It remains to prove the following.

Lemma 1.3. Let $\Sigma=\bigcup_{i=1}^{m}\left(\{i\} \times J_{i}\right)$ be a support set for $\Pi_{m, n}$. Then the bipartite graph $G(\Sigma)$ is connected.

Proof. Using condition (0.1) for $p=m$, we see that every column index $j \in[n]$ belongs to some $J_{i}$, i.e., is joined by an edge in $G(\Sigma)$ with some $i \in[m]$. Therefore, $G(\Sigma)$ can be disconnected only if there is a proper subset $I \subset[m]$ such that no point in $I$ is connected by a path in $G(\Sigma)$ with some point in $\bar{I}$. This means that the subsets $\bigcup_{i \in I} J_{i}$ and $\bigcup_{i \in I} J_{i}$ are disjoint to each other. However, this contradicts 
$(0.1)$ because

$$
\begin{aligned}
\operatorname{card}\left(\bigcup_{i \in I} J_{i}\right)+\operatorname{card}\left(\bigcup_{i \in I} J_{i}\right) & \geq(n-m+\operatorname{card}(I))+(n-m+\operatorname{card}(\bar{I})) \\
& =2 n-m>n .
\end{aligned}
$$

Lemma 1.3 and Theorem 1.1 are proven.

\section{The Simplicity Criterion: Attractors and Circuits}

In this section we give a simplicity criterion for vertices of $\Pi_{m, n}$. We start with an almost obvious general criterion and then specialize it for our situation.

Let $P$ be a polytope in some affine subspace of $\mathbf{R}^{N}$, and let $v$ be a vertex of $P$. Let $E \subset \mathbf{R}^{N}$ be a vector subspace of dimension $M$ containing all edges of $P$. Let $p o s(v-P) \subset E$ denote the cone generated by all vectors $v-v^{\prime}$ for $v^{\prime} \in P$, and $N_{v}(P) \subset E^{*}$ be the normal cone of $P$ at $v$, i.e., $N_{v}(P)=\left\{\varphi \in E^{*}: \varphi\left(v-v^{\prime}\right) \geq 0\right.$ for $\left.v^{\prime} \in P\right\}$ (so the cones $p o s(v-P)$ and $N_{v}(P)$ are dual to each other).

Proposition 2.1. Suppose $\left\{\varphi_{1}, \ldots, \varphi_{M}\right\}$ is a basis of $E^{*}$ such that all forms $\varphi_{i}$ belong to $N_{v}(P)$, and all vectors $e_{1}, \ldots, e_{M}$ in the dual basis of $E$ belong to pos $(v-P)$. Then $\operatorname{dim}(P)=M, v$ is a simple vertex of $P$, the facets of $P$ containing $v$ are parallel to the hyperplanes $\left\{\varphi_{1}=0\right\}, \ldots,\left\{\varphi_{M}=0\right\}$, and the edges of $P$ containing $v$ are parallel to the vectors $e_{1}, \ldots, e_{M}$.

Proof. Let $C \subset E$ be the cone generated by $e_{1}, \ldots, e_{M}$, and let $C^{\prime} \subset E^{*}$ be the cone generated by $\varphi_{1}, \ldots, \varphi_{M}$. Since the bases are dual, the cones $C$ and $C^{\prime}$ are dual to each other. By the assumptions, $C \subset \operatorname{pos}(v-P)$ and $C^{\prime} \subset N_{v}(P)$. On the other hand, passing to dual cones reverses inclusions, so $N_{v}(P)=\operatorname{pos}(v-P)^{*} \subset$ $C^{*}=C^{\prime}$ and $\operatorname{pos}(v-P)=N_{v}(P)^{*} \subset C^{*}=C$. We conclude that $C=\operatorname{pos}(v-P)$ and $C^{\prime}=N_{v}(P)$, which gives all our statements.

We apply Proposition 2.1 for $P=\Pi_{m, n}, E \subset \mathbf{R}^{m \times n}$ is the subspace of matrices with zero row and column sums, and $v$ is a vertex of $\Pi_{m, n}$ encoded by a coherent matching field $\Lambda=\left(\Lambda_{\sigma}\right)$. Therefore, in our situation $M=(m-1)(n-1)$. For any two subsets $I \subset[m], J \subset[n]$ we define $\varphi_{I J} \in E^{*}$ by $\varphi_{I J}=\sum_{i \in I, j \in J} \varphi_{i j}$ (the forms $\varphi_{i j}$ were defined in Section 1). Since $\Pi_{m, n}$ consists of matrices with nonnegative entries, $-\varphi_{i j} \in N_{v}\left(\Pi_{m, n}\right)$ for all $(i, j) \notin \operatorname{supp}(v)$. In all our examples the family $\left\{\varphi_{1}, \ldots, \varphi_{M}\right\}$ from Proposition 2.1 consists of $m(m-1)$ forms of the type $-\varphi_{i j}$ for $(i, j) \notin \operatorname{supp}(v)$ and of $(m-1)(n-m-1)$ forms of the type $\varphi_{I J}$ for some balanced pairs $(I, J)$ (see the Introduction for the definition of balanced pairs).

Let us reformulate the condition $\varphi_{I J} \in N_{v}\left(\Pi_{m, n}\right)$ in combinatorial terms. We say that a pair $(I, J)$ is an attractor for a matching field $\Lambda=\left(\Lambda_{\sigma}\right)$ if for each $m$-element subset $\sigma \subset[n]$ we have $I \subset \Lambda_{\sigma}(\sigma \cap J)$ or $\Lambda_{\sigma}(\sigma \cap J) \subset I$ (depending on which set is smaller). 
Proposition 2.2. The form $\varphi_{I J}$ belongs to $N_{v(\Lambda)}\left(\Pi_{m, n}\right)$ if and only if $(I, J)$ is an attractor for $\Lambda$.

Proof. The inclusion $\varphi_{I J} \in N_{v(\Lambda)}\left(\Pi_{m, n}\right)$ means that the sum of matrix entries $\sum_{i \in I, j \in J} x_{i j}$ attains its maximum on $\Pi_{m, n}$ at $v(\Lambda)$. The contribution of a matching $\Lambda_{\sigma}^{\prime}$ to this sum is not greater than $\min (\operatorname{card}(I), \operatorname{card}(\sigma \cap J))$, and it attains this value precisely when $I \subset \Lambda_{\sigma}^{\prime}(\sigma \cap J)$ or $\Lambda_{\sigma}^{\prime}(\sigma \cap J) \subset I$. This proves our proposition.

The proof of Proposition 2.2 allows us to refine it as follows.

Proposition 2.2'. Let $\operatorname{card}(I)=k, \operatorname{card}(J)=r$. The maximum value of the form $\sum_{i \in I, j \in J} x_{i j}$ on $\Pi_{m, n}$ is equal to $b_{k r}$ given by $(0.3)$, and it is attained at a vertex $v(\Lambda)$ if and only if $(I, J)$ is an attractor for $\Lambda$.

Proof. It is enough to observe that

$$
b_{k r}=\sum_{\sigma} \min (\operatorname{card}(I), \operatorname{card}(\sigma \cap J)),
$$

the sum over all $m$-element subsets of $[n]$.

We close this section by recalling the description of edges of $\Pi_{m, n}$ given in [7]. For our present purposes we reformulate it as follows. By a circuit matrix we mean a matrix of the form $C=\Lambda_{\sigma}-\Lambda_{\sigma}^{\prime}$, where $\Lambda_{\sigma}$ and $\Lambda_{\sigma}^{\prime}$ are two different matchings with the same support $\sigma$, such that the permutation $\Lambda_{a}^{-1} \circ \Lambda_{\sigma}^{\prime}$ of $[m]$ has only one cycle of length $>1$ in its cycle decomposition.

Proposition 2.3. Let $v(\Lambda)$ be a vertex of $\Pi_{m, n}$.

(a) For every m-element subset $\sigma \subset[n]$ and every matching $\Lambda_{\sigma}^{\prime} \neq \Lambda_{\sigma}$ with support $\sigma$ the matrix $\Lambda_{\sigma}-\Lambda_{\sigma}^{\prime}$ lies in the cone $\operatorname{pos}\left(v(\Lambda)-\Pi_{m, n}\right)$.

(b) If $v\left(\Lambda^{\prime}\right)$ is a vertex of $\Pi_{m, n}$ adjacent to $v(\Lambda)$, then the edge $v(\Lambda)-v\left(\Lambda^{\prime}\right)$ has the form $d C$ for some positive integer $d$ and some circuit $C$ of the form $\Lambda_{\sigma}-\Lambda_{\sigma}^{\prime}$.

This is essentially proven in Proposition 1.9 of [7].

\section{Greedy Supports and Greedy Matching Fields}

Here we introduce the greedy algorithm for constructing a coherent matching field with a given support set. This algorithm does not always work, and we characterize the support sets for which it does. 
Let $J_{1}, \ldots, J_{m}$ be subsets of $[n]$ of the same cardinality $n-m+1$. The greedy matching field with support $\left(J_{1}, \ldots, J_{m}\right)$ associates to each $m$-element subset $\sigma \subset[n]$ the matching $\Lambda_{\sigma}=\left\{\left(1, j_{1}\right), \ldots,\left(m, j_{m}\right)\right\}$ constructed by the following greedy algorithm:

$$
\begin{aligned}
& j_{1}:=\min \left(\sigma \cap J_{1}\right), \\
& j_{2}:=\min \left(\left(\sigma \backslash\left\{j_{1}\right\}\right) \cap J_{2}\right), \\
& \quad \vdots \\
& j_{m}:=\min \left(\left(\sigma \backslash\left\{j_{1}, j_{2}, \ldots, j_{m-1}\right\}\right) \cap J_{m}\right) .
\end{aligned}
$$

We say that $\left(J_{1}, \ldots, J_{m}\right)$ is greedy if the greedy algorithm is well defined, i.e., for each $k=1, \ldots, m$ we have

$$
\left(\sigma \backslash\left\{j_{1}, j_{2}, \ldots, j_{k-1}\right\}\right) \cap J_{k} \neq \varnothing
$$

Proposition 3.1. Every greedy matching field is coherent, i.e., defines a vertex of $\Pi_{m, n}$.

Proof. Clearly, a greedy matching field is lexicographic in the sense of Example 1.2 of [7], which implies that it is coherent.

Proposition 3.1 implies that if $\left(J_{1}, \ldots, J_{m}\right)$ is greedy, then $\Sigma=\bigcup_{i=1}^{m}\left(\{i\} \times J_{i}\right)$ is a support set for $\Pi_{m, n}$. Note that Proposition 3.1 is a consequence of the following more general statement whose proof is left to the reader.

Proposition 3.1'. For every support set $\Sigma$ the lexicographically first matching field with support $\Sigma$ is coherent.

Proposition 3.2. The following conditions on a collection $\left(J_{1}, \ldots, J_{m}\right)$ are equivalent:

(1) $\left(J_{1}, \ldots, J_{m}\right)$ is greedy.

(2) $\min \left(J_{k}\right) \notin J_{l}$ for $1 \leq k<l \leq m$.

Proof. We denote $\mu_{k}=\min \left(J_{k}\right)$ and $\bar{J}_{k}=[n] \backslash J_{k}$. First suppose that (2) does not hold, and let $k$ be minimal such that $\mu_{k} \in J_{l}$ for some $l>k$. Take $\sigma=\left\{\mu_{k}\right\} \cup \bar{J}_{l}$. By our choice of $k$, all indices $\mu_{1}, \ldots, \mu_{k-1}$ are distinct and lie in $\vec{J}_{l}$. Therefore, the greedy algorithm for $\sigma$ will produce $j_{i}=\mu_{i}$ for $i=1, \ldots, k$. However, then it will fail to produce $j_{l}$ because $\sigma \backslash\left\{\mu_{1}, \mu_{2}, \ldots, \mu_{k}\right\} \subset \bar{J}_{l}$.

Now suppose that (2) is true, i.e., $\left\{\mu_{1}, \mu_{2}, \ldots, \mu_{k-1}\right\} \subset \bar{J}_{k}$ for $k=1, \ldots, m$. Suppose the greedy algorithm for some $\sigma$ produces $j_{1}, j_{2}, \ldots, j_{k-1}$ but fails to produce $j_{k}$. This means that $\sigma^{\prime}:=\sigma \backslash\left\{j_{1}, j_{2}, \ldots, j_{k-1}\right\}$ is a subset of $\bar{J}_{k}$ of cardinality $m-k+1=(m-1)-(k-2)$. Then $\mu_{i} \in \sigma^{\prime}$ for some $i=1, \ldots, k-1$. However, this contradicts the following lemma. 
Lemma 3.3. If $\mu_{i}=\min \left(J_{i}\right) \in \sigma$ for some $i$, then the greedy algorithm for $\sigma$ will select $\mu_{i}$ as one of $j_{1}, \ldots, j_{i}$.

Proof of Lemma 3.3. If $\mu_{i} \notin\left\{j_{1}, j_{2}, \ldots, j_{i-1}\right\}$, then

$$
\mu_{i}=\min \left(\left(\sigma \backslash\left\{j_{1}, j_{2}, \ldots, j_{i-1}\right\}\right) \cap J_{i}\right)
$$

so it will be selected as $j_{i}$.

Proposition 3.2 is proven.

For the rest of this section let $\Lambda=\left(\Lambda_{\sigma}\right)$ be the greedy matching field with support $\left(J_{1}, \ldots, J_{m}\right)$, and $\mu_{k}=\min \left(J_{k}\right)$ for $k=1, \ldots, n$. We investigate balanced attractors for $\Lambda$ lying in several first rows.

Proposition 3.4. Let $1 \leq k \leq m-1,1 \leq l \leq n-m-1$, and suppose $J \subset[n]$ is a subset of cardinality $k+l$ such that $([k], J)$ is an attractor for $\Lambda$. Then

$$
J=\left\{\mu_{1}, \ldots, \mu_{k-1}\right\} \cup\left\{(l+1) \text { first minimal elements of } J_{k}\right\}
$$

Proof. We have to prove three statements:

(1) $\left\{\mu_{1}, \ldots, \mu_{k-1}\right\} \subset J$.

(2) $J \backslash\left\{\mu_{1}, \ldots, \mu_{k-1}\right\} \subset J_{k}$.

(3) If $j, j^{\prime} \in J_{k}, j<j^{\prime}$, and $j^{\prime} \in J$, then $j \in J$.

All of them are proven by contradiction.

Proof of (1). Suppose $\mu_{i} \notin J$ for some $i \in[k-1]$. Since $k \leq \operatorname{card}(J)$, and $m-1-k \leq n-1-\operatorname{card}(J)=\operatorname{card}\left(\bar{J}\left\{\mu_{i}\right\}\right)$, we can choose an $m$-element subset $\sigma \subset[n]$ so that $\operatorname{card}(\sigma \cap J)=k$ and $\mu_{i} \in \sigma$. By Lemma $3.3, \Lambda_{\sigma}\left(\mu_{i}\right) \in[k]$ so $[k] \not \subset$ $\Lambda_{\sigma}(\sigma \cap J)$. Hence $([k], J)$ is not an attractor.

Proof of (2). Suppose $\left(J \backslash\left\{\mu_{1}, \ldots, \mu_{k-1}\right\}\right) \cap \bar{J}_{k} \neq \varnothing$. Since $\left\{\mu_{1}, \ldots, \mu_{k-1}\right\} \subset \bar{J}_{k}$ by Proposition 3.2, it follows that $\operatorname{card}\left(J \cap \bar{J}_{k}\right) \geq k$. Take $\sigma=\bar{J}_{k} \cup\{j\}$ for some $j \in J_{k} \backslash J$. Then card $(\sigma \cap J) \geq k$ but $[\mathrm{k}] \not \subset \Lambda_{\sigma}(\sigma \cap J)$ because $k$ can be only equal to $\Lambda_{\sigma}(j)$. Hence $([k], J)$ is not an attractor.

Proof of (3). Suppose $j, j^{\prime} \in J_{k}, j<j^{\prime}, j^{\prime} \in J$ but $j \notin J$. Take

$$
\sigma=\left\{j, j^{\prime}, \mu_{1}, \ldots, \mu_{k-1}\right\} \cup \sigma^{\prime}
$$

where $\sigma^{\prime}$ is some ( $m-1-k$ )-element subset of $\widetilde{J}_{k} \backslash\left\{\mu_{1}, \ldots, \mu_{k-1}\right\}$. Using the greedy algorithm, we see that $\Lambda_{\sigma}$ contains $\left\{\left(1, \mu_{1}\right),\left(2, \mu_{2}\right), \ldots,\left(k-1, \mu_{k-1}\right),(k, j)\right\}$. As before, we have $\operatorname{card}(\sigma \cap J) \geq k$ but $[k] \not \subset \Lambda_{\sigma}(\sigma \cap J)$, hence $([k], J)$ is not an attractor.

These three contradictions complete the proof of Proposition 3.4. 
We write $J_{k}=\left\{\mu_{k 1}, \mu_{k 2}, \ldots, \mu_{k, n-m+1}\right\}$, where $\mu_{k 1}<\mu_{k 2}<\cdots<\mu_{k, n-m+1}$; in particular, $\mu_{k 1}=\mu_{k}$. Let

$$
J(k, l)=\left\{\mu_{11}, \mu_{21}, \ldots, \mu_{k-1,1}, \mu_{k 1,}, \mu_{k 2}, \ldots, \mu_{k, l+1}\right\}
$$

Proposition 3.4 motivates the following definition. We say that a greedy support $\left(J_{1}, \ldots, J_{m}\right)$ is attractive if all pairs $([k], J(k, l))$ for $1 \leq k \leq m-1,1 \leq l \leq$ $n-m-1$ are attractors for the greedy matching field with support $\left(J_{1}, \ldots, J_{m}\right)$.

Proposition 3.5. A greedy support $\left(J_{1}, \ldots, J_{m}\right)$ is attractive if and only if

$$
\mu_{k-1, l+1} \in\left\{\mu_{k l}, \mu_{k, l+1}\right\} \quad \text { for } \quad 2 \leq k \leq m-1, \quad 1 \leq l \leq n-m-1
$$

Proof. Suppose that (3.1) does not hold, and let $(k, l)$ be its lexicographically first violation. Then $\left\{\mu_{k-1,2}, \mu_{k-1,3}, \ldots, \mu_{k-1, l}\right\} \subset\left\{\mu_{k 1}, \mu_{k 2}, \ldots, \mu_{k, l}\right\}$, so

$$
\left\{\mu_{k 1}, \mu_{k 2}, \ldots, \mu_{k, l}\right\} \backslash\left\{\mu_{k-1,2}, \mu_{k-1,3}, \ldots, \mu_{k-1, l}\right\}=\left\{\mu^{\prime}\right\}
$$

for some index $\mu^{\prime}$. We choose an $m$-element subset $\sigma \subset[n]$ in such a way that $\sigma \ni \mu_{1}, \mu_{2}, \ldots, \mu_{k-2}, \mu_{k-1, l+1}, \mu^{\prime}, \mu_{k, l+1}$ but $\sigma$ does not contain any of the elements $\mu_{k-1,1}, \mu_{k-1,2}, \ldots, \mu_{k-1, l}$; clearly, the restrictions on $k$ and $l$ make such a choice possible. Then $\sigma \cap J(k, l)=\left\{\mu_{1}, \mu_{2}, \ldots, \mu_{k-2}, \mu^{\prime}, \mu_{k, l+1}\right\}$ has cardinality $k$. Using the greedy algorithm, we see that the matching $\Lambda_{\sigma}$ contains

$$
\left\{\left(1, \mu_{1}\right),\left(2, \mu_{2}\right), \ldots,\left(k-2, \mu_{k-2}\right),\left(k-1, \mu_{k-1, l+1}\right),\left(k, \mu^{\prime}\right)\right\}
$$

Since $\mu_{k-1, l+1} \notin J(k, l)$, it follows that $\Lambda_{\sigma}(J(k, l)) \neq[k]$, hence $([k], J(k, l))$ is not an attractor for $\Lambda$. This proves the "only if" part of the proposition.

To prove the "if" part we use the following lemma.

Lemma 3.6. Suppose that after $k-1$ steps of the greedy algorithm we have $\left(\sigma \backslash\left\{j_{1}, \ldots, j_{k-1}\right\}\right) \cap J(k, l) \neq \varnothing$. Then $j_{k} \in \sigma \cap J(k, l)$.

Proof of Lemma 3.6. By Lemma 3.3, $\sigma \backslash\left\{j_{1}, \ldots, j_{k-1}\right\}$ does not contain any of $\mu_{1}, \ldots, \mu_{k-1}$. Therefore, the intersection $\left(\sigma \backslash\left\{j_{1}, \ldots, j_{k-1}\right\}\right) \cap\left\{\mu_{k 1}, \mu_{k 2}, \ldots, \mu_{k, l+1}\right\}$ is not empty. However, then the greedy algorithm for $\sigma$ selects an element of this intersection as $j_{k}$.

Now suppose that (3.1) holds. To prove that all pairs $([k], J(k, l))$ for

$$
1 \leq k \leq m-1,1 \leq l \leq n-m-1
$$


are attractors for $\Lambda$ we use induction on $k$. For $k=1$ the statement follows from Lemma 3.6. So we can assume that $k \geq 2$, and that $([k-1], J(k-1, l)$ is an attractor for $\Lambda$. Notice that, by (3.1), $J(k-1, l) \subset J(k, l)$. Consider two cases.

(a) $\operatorname{card}(\sigma \cap J(k, l)) \geq k$. Then $\operatorname{card}(\sigma \cap J(k-1, l)) \geq k-1$, so by induction $j_{1}, \ldots, j_{k-1} \in \sigma \cap J(k-1, l) \subset \sigma \cap J(k, l)$. By Lemma 3.6, $j_{k} \in \sigma \cap J(k, l)$, hence $([k], J(k, l))$ is an attractor for $\Lambda_{\sigma}$.

(b) $\operatorname{card}(\sigma \cap J(k, l)) \leq k-1$. Then $\operatorname{card}(\sigma \cap J(k-1, l)) \leq k-1$, so by induction $\sigma \cap J(k-1, l) \subset\left\{j_{1}, \ldots, j_{k-1}\right\}$. If $\sigma \cap J(k, l) \subset\left\{j_{1}, \ldots, j_{k-1}\right\}$, then there is nothing to prove. If $\left(\sigma \cap J(k, l) \backslash\left\{j_{1}, \ldots, j_{k-1}\right\}=\left\{\mu^{\prime}\right\}\right.$, then by Lemma $3.6 j_{k}=\mu^{\prime}$, and we are done again.

\section{Vertices of Diagonal Type}

In this section we introduce a class of vertices of diagonal type of $\Pi_{m, n}$ and describe the cone of $\Pi_{m, n}$ at every such vertex. We fix a sequence $c=\left(c_{1}, \ldots, c_{m-1}\right)$ of distinct indices in $[n]$, and consider the subsets $J_{i}=J_{i}(c), i \in[m]$, given by $(0.2)$. Notice that by construction we have

$$
J_{k} \backslash J_{k+1}=\left\{\mu_{k}\right\}=\left\{\min J_{k}\right\}, \quad J_{k+1} \backslash J_{k}=\left\{c_{k}\right\} .
$$

We call a family of type $\left(J_{1}(c), \ldots, J_{m}(c)\right)$ for some $c$ a support of diagonal type.

Proposition 4.1. A family $\left(J_{1}(c), \ldots, J_{m}(c)\right)$ is an attractive greedy support.

Proof. The greedy property follows at once from the criterion given by Proposition 3.2(2). In particular, this implies that $\left(J_{1}(c), \ldots, J_{m}(c)\right)$ defines a support set for $\Pi_{m, n}$. The attractiveness condition (3.1) follows at once from (4.1); in fact, (4.1) implies a stronger statement:

$$
\mu_{k-1, l+1} \in\left\{\mu_{k l}, \mu_{k, l+1}\right\} \quad \text { for } \quad 2 \leq k \leq m, 1 \leq l \leq n-m .
$$

Let $\Lambda=\Lambda(c)$ be the greedy matching field with support $\left(J_{1}(c), \ldots, J_{m}(c)\right)$. By Proposition 3.1, $\Lambda$ is coherent, i.e., defines the vertex $v(\Lambda)=v(c)$ of $\Pi_{m, n}$. We call $\Lambda$ a matching field of diagonal type, and $v(c)$ a vertex of diagonal type.

In the remainder of this section we freely use the notation of Sections 2 and 3.

Theorem 4.2. The normal cone $N_{v(c)}\left(\Pi_{m, n}\right)$ is a simplicial cone of dimension $(m-1)(n-1)$ generated by linear forms $-\varphi_{i j}$, for $i \in[m], j \in \bar{J}_{i}$, and $\varphi_{[k], J(k, l)}$, for $k \in[m-1], l \in[n-m-1]$.

This theorem has two important corollaries.

Corollary 4.3. The forms $\varphi_{[k], J(k, l)}$ for $k \in[m-1], l \in[n-m-1]$ generate the cone $N_{v(c)}\left(\Pi_{m, n}\left(J_{1}, \ldots, J_{m}\right)\right)$. 
Corollary 4.4. The vertex $v(c)$ is simple for both polytopes $\Pi_{m, n}$ and

$$
\Pi_{m, n}\left(J_{1}(c), \ldots, J_{m}(c)\right)
$$

We have $\operatorname{dim}\left(\Pi_{m, n}\right)=(m-1)(n-1)$ and

$$
\operatorname{dim}\left(\Pi_{m, n}\left(J_{1}(c), \ldots, J_{m}(c)\right)=(m-1)(n-m-1) .\right.
$$

Proof of Theorem 4.2. First we prove that the linear forms $-\varphi_{i j}$, for $i \in[m]$, $j \in \bar{J}_{i}$, and $\varphi_{[k], J(k, l)}$, for $k \in[m-1], l \in[n-m-1]$, form a basis of $E^{*}$. Consider the subset of matrix indices

$$
\begin{aligned}
T= & \left\{\left(1, \mu_{1}\right),\left(2, \mu_{2}\right), \ldots,\left(m-1, \mu_{m-1}\right),\left(1, \mu_{1, n-m+1}\right),\left(2, \mu_{2, n-m+1}\right), \ldots,\right. \\
& \left.\left(m-1, \mu_{m-1, n-m+1}\right),\left(m, \mu_{m 1}\right),\left(m, \mu_{m 2}\right), \ldots,\left(m, \mu_{m, n-m+1}\right)\right\} .
\end{aligned}
$$

Lemma 4.5. The bipartite graph $G(T)$ (see Section 1) is a spanning tree.

Proof of Lemma 4.5. Let us rearrange column indices in the following order: $\mu_{1}, \mu_{2}, \ldots, \mu_{m-1}, \mu_{m 1}, \mu_{m 2}, \ldots, \mu_{m, n-m+1}$. The transformed graph $G(T)$ has the edges $(1,1),(2,2), \ldots,(m-1, m-1),(m, r)$ for $m \leq r \leq n$, and $\left(1, s_{1}\right),\left(2, s_{2}\right), \ldots$, $\left(m-1, s_{m-1}\right)$, where the $s_{i}$ are some indices such that $s_{i}>i$. Such a graph is obviously connected. Since the number of edges is

$$
(m-1)+(m-1)+(n-m+1)=m+n-1,
$$

the graph $G(T)$ is a spanning tree.

By Lemma 1.2, the forms $\left\{\varphi_{i j}:(i, j) \in \bar{T}\right\}$ form a basis in $E^{*}$. Let us label our system of forms by the same set $\bar{T}$ by setting $y_{i j}=-\varphi_{i j}$ for $j \in \bar{J}_{i}$, and $y_{k j}=\varphi_{[k], J(k, l)}$ for $j=\mu_{k, l+1}, k \in[m-1], l \in[n-m-1]$. Consider a linear ordering $\prec$ of $\bar{T}$ such that the indices $(i, j)$ with $j \in \bar{J}_{i}$ go first (in arbitrary order), and the remaining indices are ordered lexicographically. The fact that the $y_{i j}$ form a basis of $E^{*}$ follows from the next lemma.

Lemma 4.6. The transition matrix from $\left\{y_{i j}:(i, j) \in \bar{T}\right\}$ to $\left\{\varphi_{i j}:(i, j) \in \bar{T}\right\}$ is triangular with all diagonal entries equal to \pm 1 , i.e., each $y_{i j}$ has the form

$$
y_{i j}= \pm \varphi_{i j}+\sum_{\left(i^{\prime}, j^{\prime}\right) \prec(i, j)} a_{i j^{\prime} j^{\prime}}^{i^{\prime}} \varphi_{i^{\prime} j^{\prime}}
$$

Proof of Lemma 4.6. It is enough to treat $y_{k j}=\varphi_{[k], J(k, l)}$. It has the required form up to a linear combination of forms $\varphi_{i, \mu_{1}}$ and $\varphi_{i, \mu_{i, n-m+1}}$ for $1 \leq i \leq k-1$. It remains to verify the following statement: if a form $\varphi_{i^{\prime} j^{\prime}}$ with $\left(i^{\prime}, j^{\prime}\right) \in \bar{T}, j^{\prime} \in J_{i^{\prime}}$, appears in the expansion of either of the forms $\varphi_{i, \mu_{t}}$ or $\varphi_{i, \mu_{1, n-m+1}}$, then $i^{\prime} \leq i$. This 
is proven by induction on $i$ with the help of two transformations: replacing $\varphi_{i, \mu_{i}, n-m+1}$ by - (sum of all the other entries in the same row), and then replacing $\varphi_{i, \mu_{i}}$ by - (sum of all the other entries in the same column).

We have shown that linear forms in Theorem 4.2 form a basis of $E^{*}$. By Propositions 2.2 and 4.1, all these forms lie in $N_{v(c)}\left(\Pi_{m, n}\right)$. Now consider the dual basis $\left\{C_{i j}:(i, j) \in \bar{T}\right\}$ in $E$ defined by

$$
y_{i^{\prime} j^{\prime}}\left(C_{i j}\right)=\delta_{(i, j),\left(i^{\prime} j^{\prime}\right)}
$$

In view of Propositions 2.1 and 2.3, Theorem 4.2 is a consequence of the following.

Proposition 4.7. Every $C_{i j}$ is a circuit matrix of the form $\Lambda_{\sigma}(c)-\Lambda_{\sigma}^{\prime}$ for some $\sigma$ and some matching $\Lambda_{\sigma}^{\prime}$.

Proof of Proposition 4.7. First consider the case $(i, j)=\left(k, \mu_{k, l+1}\right)$ with $k \in[m-1], l \in[n-m-1]$. Let $j^{\prime}=\mu_{k, l+2}$. We claim that

$$
C_{k j}=E_{k j}-E_{k j^{\prime}}-E_{k+1, j}+E_{k+1, j^{\prime}}
$$

where $E_{k j}$ is a matrix unit. Equality (4.3) is proven by inspection (we need the fact that both $j$ and $j^{\prime}$ belong to $J_{k+1}$ but this follows from (4.2)). To show that our proposition is true for $C_{k j}$ we need to include $\left\{j, j^{\prime}\right\}$ into an $m$-element subset $\sigma \subset[n]$ such that $\left\{(k, j),\left(k+1, j^{\prime}\right)\right\} \subset \Lambda_{\sigma}$. Choose $\sigma$ so that it contains $\left\{\mu_{1}, \ldots, \mu_{k-1}\right\}$, and $\sigma \cap\left\{\mu_{k, 1}, \mu_{k+1,1}, \mu_{k+1,2}, \ldots, \mu_{k+1, l+2}\right\}=\left\{j, j^{\prime}\right\}$. Using the greedy algorithm, we see that $\Lambda_{\sigma}$ contains

$$
\left\{\left(1, \mu_{1}\right),\left(2, \mu_{2}\right), \ldots,\left(k-1, \mu_{k-1}\right),(k, j),\left(k+1, j^{\prime}\right)\right\}
$$

as required.

Now consider $C_{k j}$ for $k \in[m], j \in \bar{J}_{k}$. Since

$$
\bar{J}_{k}=\left\{\mu_{1}, \mu_{2}, \ldots, \mu_{k-1}, c_{k}, c_{k+1}, \ldots, c_{m-1}\right\}
$$

(see (0.2)), we need to consider two cases:

(1) Suppose $j=\mu_{i}$ for some $i<k$. Consider the sequence $i=i(1)<i(2)<\cdots<$ $i(t)<i(t+1)=k$, where $i(v+1)$ for $v=1, \ldots, t-1$ is defined by the conditions $i(v+1)<k$ and $\mu_{i(v+1), 1}=\mu_{i(v), 2}$. The process terminates for $v=t$ such that $i(v+1)$ satisfying the two conditions above does not exist; by (4.2), this happens exactly when $\mu_{i(t), 2} \in J_{k}$. Denote $j(v)=\mu_{i(v), 1}$ for $v=1, \ldots, t$, and $j(t+1)=\mu_{i(t), 2}$. Then $C_{k j}$ is given by the formula

$$
C_{k j}=-E_{k j}+\sum_{v=1}^{t}\left(E_{i(v), j(v)}-E_{i(v), j(v+1)}\right)+E_{k, j(t+1)} .
$$


Formula (4.3) is verified by inspection. To show that our proposition is true for $C_{k j}$ we have to find an $m$-element subset $\sigma \subset[n]$ such that $\Lambda_{\sigma}$ contains $(i(v)$, $j(v))$ for $v=1, \ldots, t+1$. We take $\sigma=\{j(t+1)\} \cup \bar{J}_{k}$ and apply the following lemma.

Lemma 4.8. If $\sigma=\{j\} \cup \bar{J}_{k}$ for some $j \in J_{k}$, then

$$
\Lambda_{\sigma}=\left\{\left(1, \mu_{1}\right),\left(2, \mu_{2}\right), \ldots,\left(k-1, \mu_{k-1}\right),(k, j),\left(k+1, c_{k}\right), \ldots,\left(m, c_{m-1}\right)\right\}
$$

This statement is an immediate consequence of the greedy algorithm.

(2) Suppose that $j=c_{l}$ for some $l$ with $k \leq l \leq m-1$. Consider the sequence $k=k(1)<k(2)<\cdots<k(t)<k(t+1)=l+1$, where $k(v+1)$ for $v=1, \ldots, t-1$ is defined as the minimal index such that $k(v)<k(v+1)<l+1$ and

$$
\mu_{k(v+1), n-m}=\mu_{k(v), n-m+1}
$$

The process terminates for $v=t$ such that $k(v+1)$ satisfying these conditions does not exist. Denote $j(v)=\mu_{k(v), n-m+1}$ for $v=1, \ldots, t$ and $j(t+1)=j$. Then $C_{k j}$ is given by the formula

$$
C_{k j}=-E_{k j}+\sum_{v=1}^{t}\left(E_{k(v) . j(v)}-E_{k(v+1), j(v)}\right)+E_{l+1, j}
$$

Formula (4.3) is again verified by inspection. To show that our proposition is true for $C_{k j}$ we have to find an $m$-element subset $\sigma \subset[n]$ such that $\Lambda_{\sigma}$ contains $(k(v), j(v))$ for $v=1, \ldots, t+1$. We take $\sigma=\{j(1)\} \cup \bar{J}_{k}$. Our statement follows from Lemma 4.8 and the observation that $j(v)=c_{k(v)}$ for $v=2, \ldots, t+1$ (this is a consequence of $(4.1)$ ).

Proposition 4.7 and Theorem 4.2 are proven.

Combining Propositions 2.1 and 4.7 we get the following.

\section{Corollary 4.9.}

(a) The edges of $\Pi_{m, n}$ containing $v(c)$ are parallel to circuit matrices given by (4.4), (4.5), and (4.6).

(b) The edges of $\Pi_{m, n}\left(J_{1}(c), \ldots, J_{m}(c)\right)$ containing $v(c)$ are parallel to circuit matrices given by (4.4).

Theorems 0.1 and 0.2 from the Introduction are immediate consequences of the results in this section. 


\section{Examples}

Example 5.1. Consider the vertex of $\Pi_{m, n}$ of diagonal type corresponding to the sequence $c=(2,3, \ldots, m)$. Using $(0.2)$, we see that $J_{i}=\{i, m+1, m+2, \ldots, n\}$ for $i=1, \ldots, m$. Let $\Gamma=\Pi_{m, n}\left(J_{1}, \ldots, J_{m}\right)$ be the corresponding support face of $\Pi_{m, n^{*}}$ The polytope $\Gamma$ is naturally identified with the secondary polytope of the product $\Delta^{m-1} \times \Delta^{n-m-1}$ of two standard simplices (see Proposition 3.11 of [7] and 3E.3 of [3]), so its vertices are in one-to-one correspondence with regular triangulations of $\Delta^{m-1} \times \Delta^{n-m-1}$. The matching field $\Lambda(c)$ is the pointed diagonal matching field considered in Example 1.4 of [7]. The vertex $v(c)$ corresponds to the standard triangulation of $\Delta^{m-1} \times \Delta^{n-m-1}$ used in algebraic topology (see, e.g., [2]).

The set $\bar{T}$ introduced in Section 4 takes the form

$$
\bar{T}=[m-1] \times(m+[n-m-1]) .
$$

By Lemma 4.5, the matrix entries $\left(x_{i j}:(i, j) \in \bar{T}\right)$ form a system of coordinates for $\Gamma$. Expressing the forms from Corollary 4.3 in terms of these coordinates, we see that the normal cone $N_{v(c)}(\Gamma)$ is generated by the forms $\psi_{k l}=\sum_{i \in[k], j \in[l]} x_{i, m+j}$ for $k \in[m-1], l \in[n-m-1]$. The edges of $\Gamma$ containing $v(c)$ are given by Corollary 4.9(b). They are parallel to vectors of the form

$$
E_{k, m+l}-E_{k, m+l+1}-E_{k+1, m+l}+E_{k+1, m+l+1} .
$$

Generally, edges of the secondary polytope correspond to flips (or restructurings) of triangulations [3, Theorem 3A.8]. Translating our edges into the language of flips we see that all possible flips of the standard triangulation consist of switching from one triangulation of a square to another one.

Example 5.1'. Let $c=\left(c_{1}, \ldots, c_{m-1}\right)$ be such that $\left\{c_{1}, \ldots, c_{m-1}\right\} \subset[m]$. Let $[m] \backslash\left\{c_{1}, \ldots, c_{m-1}\right\}=\left\{c_{0}\right\}$. Then $(0.2)$ gives $J_{i}=\left\{c_{i-1}, m+1, m+2, \ldots, n\right\}$ for $i=1, \ldots, m$. This support and the vertex $v(c)$ are obtained from those in the previous example by a permutation of columns $1,2, \ldots, m$.

Example 5.2. Let $c=(n-m+2, n-m+3, \ldots, n)$. Using $(0.2)$, we see that $J_{i}=\{i, i+1, i+2, \ldots, i+n-m\}$ for $i=1, \ldots, m$. The matching field $\Lambda(c)$ is the diagonal matching field considered in Example 1.3 of [7]. The edges of $\Pi_{m, n}$ containing $v(c)$ and lying on its support face are given by Corollary 4.10. They are parallel to vectors of the form $E_{k, k+l}-E_{k, k+l+1}-E_{k+1, k+l}+E_{k+1, k+l+1}$ for $k \in[m-1], l \in[n-m-1]$.

Example 5.3. Let $T_{m, n}$ and $S_{m, n}$ be polytopes as considered at the end of the Introduction. The polytope $\Pi_{2, n}$ is the permutohedron, and it is well known that 
$\Pi_{2, n}=S_{2, n}$ (see, e.g., [9]). Every vertex of $\Pi_{2, n}$ can be made a vertex of diagonal type by a permutation of rows and columns, since all the vertices of $\Pi_{2, n}$ form one symmetry class. This gives one more proof of the well-known statement that $\Pi_{2, n}$ is simple.

Example 5.4. It was proven in [7] (see also [5]) that $\Pi_{m, m+1}=S_{m, m+1}=T_{m, m+1}$, and this polytope is simple. All its vertices are very simple (see the Introduction). Nevertheless, in this case not every support can be transformed into a support of diagonal type by a permutation of rows and columns.

Example 5.5. Consider the polytope $\Pi_{3,5}$. It was shown in Example 3.12 of [7] that the vertices of $\Pi_{3,5}$ form six symmetry classes. We use the notation of [7], where these classes were denoted by the letters $A, B, C, D, E$, and $P$. The diagonal vertex $v(4,5)$ (see Example 5.2) belongs to the class $D$, and the pointed diagonal vertex $v(2,3)$ (Example 5.1) belongs to the class $P$. The direct calculation shows that the vertex of diagonal type corresponding to $c=(3,5)$ has the form

$$
v(3,5)=\left(\begin{array}{ccccc}
6 & 3 & 0 & 1 & 0 \\
0 & 3 & 5 & 2 & 0 \\
0 & 0 & 1 & 3 & 6
\end{array}\right)
$$

and belongs to the class $A$. It can be shown that three remaining classes $B, C$, and $E$ do not contain vertices of diagonal type. However, a vertex can be chosen in each of them whose matching field is lexicographically first for its support set. These choices are:

$$
\begin{array}{ll}
\text { Type B: } & \left(\begin{array}{lllll}
6 & 3 & 0 & 0 & 1 \\
0 & 2 & 6 & 2 & 0 \\
0 & 1 & 0 & 4 & 5
\end{array}\right), \\
\text { Type C: } & \left(\begin{array}{lllll}
5 & 4 & 1 & 0 & 0 \\
0 & 2 & 5 & 3 & 0 \\
1 & 0 & 0 & 3 & 6
\end{array}\right), \\
\text { Type E: } & \left(\begin{array}{lllll}
5 & 4 & 0 & 0 & 1 \\
0 & 2 & 6 & 2 & 0 \\
1 & 0 & 0 & 4 & 5
\end{array}\right) .
\end{array}
$$

The equations of simple facets from Theorem 0.1 take the form $x_{i, j}+x_{i, j^{\prime}}=9$ for all possible choices of $i, j$, and $j^{\prime}$. Using the results of Section 2 , it is easy to see that all these vertices are very simple. Therefore, $\Pi_{3,5}$ is simple. 
Example 5.6. Consider the polytope $\Pi_{3,6}$. As shown in Proposition 3.13 of [7], it is not simple. However, we will show that every support face of $\Pi_{3,6}$ contains a very simple vertex. By Example 3.12 of [7], there are four symmetry classes of support faces of $\Pi_{3,6}$. They were referred to in [7] as types $1-4$. The support face of type 1 contains the pointed diagonal vertex, and the support face of type 2 contains the diagonal vertex. It can be shown that the remaining two types do not contain vertices of diagonal type. Permuting rows and columns, we can choose support sets of these types so that vertices given by their lexicographically first matching fields have the form:

$$
\begin{aligned}
& \text { Type 3: } \quad\left(\begin{array}{rrrrrr}
10 & 6 & 3 & 1 & 0 & 0 \\
0 & 4 & 6 & 0 & 7 & 3 \\
0 & 0 & 1 & 9 & 3 & 7
\end{array}\right), \\
& \text { Type 4: } \quad\left(\begin{array}{rrrrrr}
9 & 7 & 3 & 0 & 0 & 1 \\
0 & 3 & 7 & 7 & 3 & 0 \\
1 & 0 & 0 & 3 & 7 & 9
\end{array}\right) .
\end{aligned}
$$

The equations of simple facets are $x_{i, j}+x_{i, j^{\prime}}=16$ and $x_{i, j}+x_{i, j^{\prime}}+x_{i, j^{\prime \prime}}=19$. Using the results of Section 2 , it is easy to see that our two vertices are very simple. This implies that all support faces of $\Pi_{3,6}$ have dimension 4 , in accordance with the general conjecture.

Example 5.7. Consider the polytope $\Pi_{4,6}$. As in the previous example, we exhibit a very simple vertex for a set of support faces representing all symmetry classes. A direct calculation along the lines of Example 3.12 of [7] shows that there are 13 different symmetry classes of support faces. In each case it is possible to choose a support face so that the lexicographically first matching field gives a very simple vertex (such a choice is not unique). Here is a list of these vertices.

$$
\begin{aligned}
& \text { Type 1: } \quad\left(\begin{array}{rrrrrr}
9 & 5 & 0 & 0 & 1 & 0 \\
0 & 5 & 8 & 2 & 0 & 0 \\
0 & 0 & 2 & 0 & 9 & 4 \\
1 & 0 & 0 & 8 & 0 & 6
\end{array}\right), \\
& \text { Type 2: } \quad\left(\begin{array}{rrrrrr}
9 & 5 & 0 & 0 & 1 & 0 \\
0 & 5 & 8 & 2 & 0 & 0 \\
0 & 0 & 2 & 8 & 0 & 5 \\
1 & 0 & 0 & 0 & 9 & 5
\end{array}\right), \\
& \text { Type 3: } \quad\left(\begin{array}{rrrrrr}
9 & 5 & 1 & 0 & 0 & 0 \\
0 & 5 & 7 & 3 & 0 & 0 \\
0 & 0 & 2 & 0 & 10 & 3 \\
1 & 0 & 0 & 7 & 0 & 7
\end{array}\right),
\end{aligned}
$$


Type 4: $\quad\left(\begin{array}{rrrrrr}10 & 4 & 0 & 0 & 1 & 0 \\ 0 & 6 & 7 & 0 & 2 & 0 \\ 0 & 0 & 3 & 9 & 0 & 3 \\ 0 & 0 & 0 & 1 & 7 & 7\end{array}\right)$,

Type 5: $\quad\left(\begin{array}{rrrrrr}10 & 4 & 0 & 1 & 0 & 0 \\ 0 & 6 & 7 & 0 & 2 & 0 \\ 0 & 0 & 2 & 9 & 0 & 4 \\ 0 & 0 & 1 & 0 & 8 & 6\end{array}\right)$,

Type 6: $\quad\left(\begin{array}{rrrrrr}10 & 4 & 1 & 0 & 0 & 0 \\ 0 & 6 & 0 & 0 & 7 & 2 \\ 0 & 0 & 9 & 0 & 2 & 4 \\ 0 & 0 & 0 & 10 & 1 & 4\end{array}\right)$,

Type 7: $\quad\left(\begin{array}{rrrrrr}10 & 4 & 0 & 0 & 0 & 1 \\ 0 & 6 & 7 & 0 & 0 & 2 \\ 0 & 0 & 3 & 9 & 0 & 3 \\ 0 & 0 & 0 & 1 & 10 & 4\end{array}\right)$,

Type 8: $\quad\left(\begin{array}{rrrrrr}10 & 4 & 1 & 0 & 0 & 0 \\ 0 & 6 & 6 & 3 & 0 & 0 \\ 0 & 0 & 3 & 6 & 6 & 0 \\ 0 & 0 & 0 & 1 & 4 & 10\end{array}\right)$,

Type 9: $\quad\left(\begin{array}{rrrrrr}10 & 0 & 4 & 0 & 0 & 1 \\ 0 & 10 & 3 & 2 & 0 & 0 \\ 0 & 0 & 3 & 7 & 5 & 0 \\ 0 & 0 & 0 & 1 & 5 & 9\end{array}\right)$,

Type 10: $\quad\left(\begin{array}{rrrrrr}10 & 0 & 4 & 1 & 0 & 0 \\ 0 & 10 & 3 & 2 & 0 & 0 \\ 0 & 0 & 3 & 0 & 9 & 3 \\ 0 & 0 & 0 & 7 & 1 & 7\end{array}\right)$,

Type 11: $\quad\left(\begin{array}{rrrrrr}10 & 0 & 4 & 1 & 0 & 0 \\ 0 & 10 & 3 & 2 & 0 & 0 \\ 0 & 0 & 3 & 6 & 0 & 6 \\ 0 & 0 & 0 & 1 & 10 & 4\end{array}\right)$, 


$$
\begin{aligned}
& \text { Type 12: } \quad\left(\begin{array}{rrrrrr}
10 & 0 & 4 & 0 & 0 & 1 \\
0 & 10 & 3 & 2 & 0 & 0 \\
0 & 0 & 3 & 7 & 0 & 5 \\
0 & 0 & 0 & 1 & 10 & 4
\end{array}\right), \\
& \text { Type 13: } \quad\left(\begin{array}{rrrrrr}
10 & 0 & 0 & 0 & 4 & 1 \\
0 & 10 & 0 & 0 & 3 & 2 \\
0 & 0 & 10 & 0 & 2 & 3 \\
0 & 0 & 0 & 10 & 1 & 4
\end{array}\right) .
\end{aligned}
$$

The equations of simple facets are $x_{i, j}+x_{i, j^{\prime}}=14$ and $\sum_{i \in I, j \in J} x_{i, j}=27$ for $\operatorname{card}(I)=2, \operatorname{card}(J)=3$. A tedious but totally routine calculation shows that all the above vertices are very simple. There are four vertices of diagonal type among them, namely, types $7,8,11$, and 13 ; they correspond, respectively, to $c=(3,4,5)$, $(4,5,6),(2,6,5)$, and $(2,3,4)$ (so the vertex of type 8 corresponds to the diagonal matching field, and that of type 13 to the pointed diagonal matching field). It is easy to show that the support faces of remaining nine types do not contain vertices of diagonal type.

Summarizing the results of the above examples we obtain the following.

Proposition 5.8. Suppose that $n \leq 6$.

(a) Every support set of $\Pi_{m, n}$ can be transformed by permutations of rows and columns into such a position that the corresponding lexicographically first matching field defines a very simple vertex.

(b) Every support face of $\Pi_{m, n}$ has dimension $(m-1)(n-m-1)$.

\section{Acknowledgments}

This work was completed during the second author's stay at the Mittag-Leffler Institute in May 1992. He gratefully acknowledges this hospitality and support. We appreciate helpful remarks and suggestions made by an anonymous referee.

\section{References}

1. D. Bernstein, A. Zelevinsky: Combinatorics of maximal minors, J. Algebra Combin., to appear.

2. P. Gabriel, M. Zisman: Calculus of Fractions and Homotopy Theory, Berlin, 1967.

3. I. M. Gelfand, M. M. Kapranov, A. V. Zelevinsky: Discriminants of polynomials in several variables and triangulations of Newton polytopes, Leningrad Math. J. 2(3) (1991), 449-505 (translated from Algebra i Analiz 2 (1990), 1-62).

4. M. M. Kapranov, B. Sturmfels, A. V. Zelevinsky: Chow polytopes and general resultants, Duke Math. J. 67(1) (1992), 189-218. 
5. V. Klee, C. Witzgall: Facets and vertices of transportation polytopes, in Mathematics of the Decision Sciences (G. Dantzig, A. Veinott, eds.), Lecture Notes in Applied Mathematics, vol. 11, American Mathematical Society, Providence, RI, pp. 257-282.

6. T. Oda: Complex Bodies and Algebraic Geometry, Springer-Verlag, New York, 1988.

7. B. Sturmfels, A. Zelevinsky: Maximal minors and their leading terms, Adv. in Math., to appear.

8. D. J. A. Welsh: Matroid Theory, Academic Press, New York, 1976.

9. V. A. Yemelichev, M. M. Kovalev, M. M. Kravtsov: Polytopes, Graphs, and Optimisation, Cambridge University Press, Cambridge, 1984.

Received July 24, 1992, and in revised form October 1, 1992. 